\title{
Triplet therapy with venetoclax, FLT3 inhibitor and decitabine for FLT3-mutated acute myeloid leukemia
}

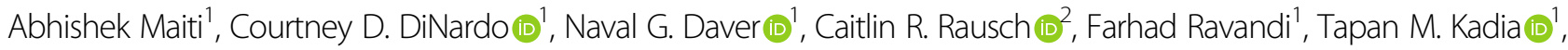 \\ Naveen Pemmaraju', Gautam Borthakur (1)', Prithviraj Bose (1)', Ghayas C. Issa (1)', Nicholas J. Short (1)', Musa Yilmaz', \\ Guillermo Montalban-Bravo', Alessandra Ferrajoli', Elias J. Jabbour', Nitin Jain', Maro Ohanian', Koichi Takahashi (i)', \\ Philip A. Thompson [1]', Sanam Loghavi $\mathbb{B}^{3}$, Kathryn S. Montalbano', Sherry Pierce', William G. Wierda', \\ Hagop M. Kantarjian (1) ${ }^{1}$ and Marina Y. Konopleva (1) ${ }^{1}$
}

FLT3 mutations occur in $20-35 \%$ patients with newly diagnosed (ND) acute myeloid leukemia (AML) and confer a higher risk of relapse and inferior overall survival (OS). Given modest benefit with first-generation multikinase inhibitors, second-generation FLT3 inhibitors (FLT3i) have been combined with low-intensity therapies (LIT) with encouraging results but are not curative ${ }^{1-4}$. Venetoclax with hypomethylating agent (HMA) has emerged as the new standard for older/unfit patients with $\mathrm{AML}^{5}$. Pre-clinical studies in $F L T 3^{\mathrm{mut}}$ cell lines, primary samples, and xenografts have shown synergy between FLT3i's and venetoclax through downregulation of Mcl-1 and $\mathrm{Bcl}-\mathrm{x}_{\mathrm{L}}{ }^{6-9}$. Clinical studies have demonstrated safety and activity of the combination of FLT3i and HMA with composite complete remission (CRc) rates of $65-80 \%$ and median OS 8.5-20 months ${ }^{1,4,10}$, as well as FLT3i and venetoclax which showed CRc rate of $85 \%$ in relapsed/ refractory $(\mathrm{R} / \mathrm{R}) F L T 3^{\text {mut }}$ AML including in patients with prior FLT3i exposure ${ }^{11}$. We hypothesized that triplet therapy combining FLT3i, venetoclax, and HMA may further improve outcomes. Hence, we added FLT3i to our regimen of 10-day decitabine with venetoclax (DEC10VEN) for $F L T 3^{\text {mut }}$ AML. We herein describe the first report of such a 'triplet' combination regimen for $F L T 3^{\text {mut }}$ AML.

\footnotetext{
Correspondence: Marina Y. Konopleva (mkonople@mdanderson.org)

${ }^{1}$ Department of Leukemia, The University of Texas MD Anderson Cancer

Center, Houston, TX, USA

${ }^{2}$ Division of Pharmacy, The University of Texas MD Anderson Cancer Center, Houston, TX, USA

Full list of author information is available at the end of the article

These authors contributed equally: Abhishek Maiti, Courtney D. DiNardo
}

This phase 2 trial (NCT03404193) enrolled ND patients with AML $>60$ years and R/R patients $>18$ years. Patients needed to have ECOG performance status $\leq 3$. Patients with favorable-risk cytogenetics and prior Bcl-2 inhibitor exposure were excluded. Patients received decitabine $20 \mathrm{mg} / \mathrm{m}^{2}$ IV for 10-days every 4-6 weeks for induction followed by decitabine for 5-days after CR/CRi, as described previously ${ }^{12}$. Venetoclax dose was $400 \mathrm{mg}$ PO daily or equivalent (with azole co-administration). Reduction of venetoclax duration to $<21$ days per cycle was permitted in cases of persistent myelosuppression, after confirming $\leq 5 \%$ blasts or hypo/acellular marrow. Addition of FLT3i of clinician's choice was allowed (Fig. S1). ND patients were admitted for the first cycle and $\mathrm{R} / \mathrm{R}$ patients were admitted for the initial venetoclax rampup. Cytoreduction to $\mathrm{WBC}<10 \times 10^{9} / \mathrm{L}$ was required prior to starting therapy and all patients received prophylaxis for tumor lysis syndrome, and antimicrobial prophylaxis.

Responses were graded per the IWG criteria for AML with adapted CRc criteria per the gilteritinib ADMIRAL and quizartinib QUANTUM-R studies ${ }^{13,14}$. The CRc included $\mathrm{CR}, \mathrm{CR}$ with incomplete platelet recovery, and $\mathrm{CR}$ with incomplete hematologic recovery ${ }^{13}$. OS was measured from start of therapy until death or censored at last follow-up. Progression-free survival was defined from the time of response until relapse, death, or censored at last follow-up. Duration of response was determined from the time of response till relapse or censored at last followup or at the time of death without relapse. Measurable residual disease (MRD) was assessed on bone marrow (BM) specimens using 8-color multiparametric flow 
cytometry (FCM) validated to a sensitivity level of $0.01-0.1 \%$. Negative results were considered valid if there had been acquisition of $\geq 200,000$ events or $\geq 200$ CD34+ myeloid precursors. A multiplex PCR-based test was used to detect FLT3-ITD or point mutations in codons 835/836 with an analytical sensitivity of $1 \%$ mutant reads in the background of wild-type reads. A targeted NGS panel was used to detect other mutations in FLT3 and co-mutations in 80 other genes with an analytical sensitivity of $5 \%$ mutant reads in a background of wild-type reads.

Between April 30, 2018 and February 10, 2020, we treated 25 patients with $F L T 3^{\text {mut }}$ AML with this triplet combination. Twelve patients had ND AML and 13 patients had R/R AML (Table 1). The median age of the ND cohort was 70 years (IQR 69-78) and the R/R cohort was 52 years (interquartile range [IQR] 35-67). Median FLT3 allelic ratio at enrollment in ND patients was 0.38 (IQR $0.17-0.45$ ) and in $R / R$ patients was 0.40 (IQR $0.32-0.52)$. The $R / R$ cohort had received a median of 2 prior lines of therapies (IQR1-3) and 8 patients (57\%) had received a prior FLT3i including sorafenib $(n=5)$, midostaurin $(n=2)$, gilteritinib $(n=1)$, and crenolanib $(n=$ 1 ) with one patient having received two prior FLT3i. Four patients (29\%) had received prior allogeneic hematopoietic stem-cell transplantation (HSCT).

FLT3i used along with DEC10-VEN in the ND cohort included gilteritinib $(n=5)$, sorafenib $(n=5)$, and midostaurin $(n=2)$, and in the R/R cohort included sorafenib $(n=5)$, gilteritinib $(n=5)$, and midostaurin $(n=3)$. Median dose and duration of FLT3i during cycle 1 for sorafenib was $400 \mathrm{mg}$ twice daily (BID; IQR 400-400) for 15 days (IQR 14-28), for midostaurin was $50 \mathrm{mg}$ BID (IQR 50-50) for 15 days (IQR 14-21) and for gilteritinib was $120 \mathrm{mg}$ daily (IQR $120-120$ ) for 14 days (IQR 14continuous). For subsequent cycles, the median dose and duration of sorafenib was $400 \mathrm{mg}$ BID (IQR 400-400) for 14 days (IQR 14-continous), for midostaurin was $50 \mathrm{mg}$ BID (IQR 50-50) daily continuously (IQR 28-continuous) and for gilteritinib was $120 \mathrm{mg}$ (IQR 80-120) daily continuously (IQR 24-continuous). Details of reductions in FLT3 inhibitor dose and venetoclax duration are mentioned in the supplement and Fig. S2. In ND patients, delay in starting subsequent cycle beyond 42 days occurred in 18 (43\%) out of 42 evaluable cycles. Among R/ $\mathrm{R}$ patients, such delay occurred in 7 (37\%) out of 19 evaluable cycles. The last ongoing cycle at the time of data cut-off was not included in this analysis.

In ND patients, the CRc rate was $92 \%$ with MRD negativity by FCM in $56 \%$ and by PCR/NGS in $91 \%$ of responders (Table 1). In R/R AML the CRc rate was $62 \%$ with MRD negativity rate by FCM in $63 \%$ and by PCR/NGS in $100 \%$ of responders. Among 8 patients with R/R AML and prior exposure to a FLT3i the CRc rate was 63\%, with FLT3 PCR negativity in 4 out of 4 responding patients tested.

Table 1 Baseline characteristics and outcomes of patients with $F L T 3^{\text {mut }}$ AML treated with FLT3 inhibitor, venetoclax, and 10-day decitabine.

\begin{tabular}{ll}
\hline $\begin{array}{l}\text { Newly } \\
\text { diagnosed AML } \\
(N=12)\end{array}$ & $\begin{array}{l}\text { Relapsed/ } \\
\text { refractory AML } \\
(N=13)\end{array}$ \\
\hline
\end{tabular}

Baseline characteristics

\begin{tabular}{|c|c|c|}
\hline Age, years & 70 [69-78] & $52[35-67]$ \\
\hline$\geq 70$ years & $6(50)$ & $2(15)$ \\
\hline Male sex & $4(33)$ & $10(77)$ \\
\hline ECOG performance status $\geq 2$ & $4(33)$ & $3(23)$ \\
\hline Peripheral blood blasts, \% & $9[3-51]$ & $58[35-70]$ \\
\hline Bone marrow blasts, $\%$ & $51[46-75]$ & $64[54-68]$ \\
\hline \multicolumn{3}{|l|}{ Diagnosis } \\
\hline De novo & $11(92)$ & $13(100)$ \\
\hline Secondary AML with AHD & $1(8)$ & $0(0)$ \\
\hline \multicolumn{3}{|l|}{ ELN 2017 risk group } \\
\hline Favorable & $5(42)$ & $5(38)$ \\
\hline Intermediate & $4(33)$ & $1(7)$ \\
\hline Adverse & $3(25)$ & $7(54)$ \\
\hline \multicolumn{3}{|l|}{ ELN 2017 cytogenetic risk } \\
\hline Favorable & $0(0)$ & $0(0)$ \\
\hline Intermediate & $12(100)$ & $9(69)$ \\
\hline Adverse & $0(0)$ & $4(31)$ \\
\hline \multicolumn{3}{|l|}{ FLT3 } \\
\hline ITD high $(\geq 0.5)$ & $1(8)$ & $4(31)$ \\
\hline ITD low $(<0.5)$ & $7(58)$ & $6(46)$ \\
\hline TKD & $3(25)$ & $1(8)$ \\
\hline ITD and TKD & $1(8)$ & $1(8)$ \\
\hline Other & $0(0)$ & $1(8)^{\mathrm{a}}$ \\
\hline \multicolumn{3}{|l|}{ Mutations } \\
\hline NPM1 & $6(50)$ & $7(54)$ \\
\hline $1 D H 1 / 2$ & $4(33)$ & $1(8)$ \\
\hline TP53 & $0(0)$ & $2(15)$ \\
\hline$R \cup N \times 1$ & $2(17)$ & $2(15)$ \\
\hline ASXL1 & $3(25)$ & $1(8)$ \\
\hline KNNRAS & $2(17)$ & $2(15)$ \\
\hline Prior therapies & 0 & $1[1-3]$ \\
\hline FLT3 inhibitor & & $8(62)$ \\
\hline Hypomethylator (HMA) & & $2(15)$ \\
\hline Intensive chemotherapy (IC) & & $12(92)$ \\
\hline Stem-cell transplantation & & $4(31)$ \\
\hline
\end{tabular}


Table 1 continued

\begin{tabular}{|c|c|c|}
\hline & $\begin{array}{l}\text { Newly } \\
\text { diagnosed AML } \\
(N=12)\end{array}$ & $\begin{array}{l}\text { Relapsed/ } \\
\text { refractory AML } \\
(N=13)\end{array}$ \\
\hline \multicolumn{3}{|l|}{ Outcomes } \\
\hline $\begin{array}{l}\text { Composite complete } \\
\text { remission rate (CRc) }\end{array}$ & $11(92)$ & $8(62)$ \\
\hline$C R$ & $9(75)$ & $3(23)$ \\
\hline CRp & $2(17)$ & $0(0)$ \\
\hline CRi & $0(0)$ & $5(38)$ \\
\hline \multicolumn{3}{|l|}{ MRD negative } \\
\hline by FCM & $5 / 9(56)$ & $5 / 8(63)$ \\
\hline by PCR/NGS & 10/11 (91) & $7 / 7(100)$ \\
\hline No response & $1(8)$ & $4(31)$ \\
\hline Aplasia & $0(0)$ & $1(8)$ \\
\hline 60-day mortality & $0(0)$ & $1(8)$ \\
\hline Time to response, months & $1.5[1.3-2.7]$ & $1.5[1.0-2.4]$ \\
\hline No. of cycles to response & $1[1-2]$ & $2[1-2]$ \\
\hline
\end{tabular}

All results expressed as no. (\%) or median [interquartile range], unless specified. $\mathrm{CR}=$ complete remission with $<5 \%$ blasts and absolute neutrophil count (ANC) $\geq 1 \times 10^{9} / \mathrm{L}$ and platelet count $\geq 100 \times 10^{9} / \mathrm{L}, \mathrm{CRp}=$ achievement of all CR criteria except for platelet recovery (platelet count $<100 \times 10^{9} / \mathrm{L}$ ), CRi $=\mathrm{CR}$ with incomplete hematologic recovery $=$ achievement of all CR criteria except for hematologic recovery with residual neutropenia (ANC $<1 \times 10^{9} / \mathrm{L}$ ) with or without RBC/platelet transfusion independence; aplasia was defined as inevaluable bone marrow sample due to cellularity $<10 \%$.

ECOG Eastern Cooperative Oncology Group, AHD antecedent hematological disorder, ELN European LeukemiaNet, MRD minimal residual disease, FCM flow cytometry, $P C R$ polymerase chain reaction, NGS next-generation sequencing.

${ }^{a}$ One patient had FLT3 S749L variant.

The 60-day mortality was $0 \%$ in ND patients and $7 \%$ $(n=1)$ in $\mathrm{R} / \mathrm{R}$ patients. There were 50 non-hematologic adverse events (AE) in 25 patients, at least possibly related to study regimen, with most frequent grade 3/4 AEs being febrile neutropenia in $40 \%$ patients $(n=10)$, infections with grade $3 / 4$ neutropenia in $36 \%$ patients $(n=9)$, infection with absolute neutrophil count (ANC) $\geq 1.0 \times$ $10^{9} / \mathrm{L}$ in $32 \%$ patients $(n=8)$, and tumor lysis syndrome in $16 \%$ patients $(n=4$, Table S1). In responding patients with ND AML, the median time to ANC recovery to $\geq 0.5 \times 10^{9} / \mathrm{L}$ after cycle 1 was 44 days and after subsequent cycles was 38 days (Fig. 1a); and median time to platelet recovery to $\geq 50 \times 10^{9} / \mathrm{L}$ after cycle 1 was 34 days and $<50 \%$ patients had platelet count drop below $50 \times$ $10^{9} / \mathrm{L}$ during subsequent cycles (Fig. 1b). In responding patients with $\mathrm{R} / \mathrm{R} A M L$, median time to ANC recovery after cycle 1 was 38 days and after subsequent cycles was 47 days. The median cycle durations in ND AML for cycle 1 and cycle 2 were 46 days (IQR 43-52) and 42 days (IQR 42-69), respectively, and in R/R AML were 32 days (IQR 30-44) and 47 days (IQR 38-61), respectively.
After a median follow-up 14.5 months (95\% CI 7.7-23.0) the median OS in ND patients was not reached with 2-year OS of $80 \%$, and in $R / R$ patients was 6.8 months (Fig. 1c). The 18-month progression-free survival in ND and $R / R$ AML was 59\% and 58\%, respectively (Fig. S3). The median duration of response was not reached in either ND or R/R patients (range 0.8-24.3 months; Fig. S4). Four ND patients underwent HSCT after response and two patients received maintenance with sorafenib $(n=1)$ and crenolanib $(n=1)$. Five $R / R$ patients underwent HSCT and one patient received maintenance with decitabine and sorafenib afterward. The 2-year OS in patients undergoing HSCT for ND and R/R patients was $100 \%$ and $53 \%$, respectively (Fig. S5). A detailed mutational landscape is shown in Fig. 1d.

Two ND patients and eight R/R patients have died. In the ND cohort, two patients died in CR/CRp due to pneumonia with grade 1 neutropenia $(n=1$, after elective discontinuation after cycle 5$)$ and unknown reason $(n=$ 1 ). Among $R / R$ patients, four patients who were refractory to this regimen died from infectious complications $(n=3)$ and of unknown reason $(n=1)$; three responding patients died of unknown reasons after relapse and one patient with aplasia died from infectious complications after HSCT. Infections contributing to death, regardless of attribution, in one ND patient and three $R / R$ patients included pneumonia due to Stenotrophomonas maltophila $(n=1)$ and unknown pathogen $(n=3)$. Among five responding patients who died, there were no deaths known to be possibly, probably, or definitely related to the study regimen. The causes of death in these five patients included pneumonia due to unknown pathogen $(n=1)$ and unknown reason $(n=4)$. Among five patients without a response who died, one death was possibly related to study regimen due to pneumonia in the setting of persistent pancytopenia and aplastic bone marrow with $<5 \%$ cellularity 16 days prior to death. Twenty patients discontinued treatment for reasons including HSCT $(n=9$, $36 \%)$, resistant disease $(n=4,16 \%)$, relapse $(n=2,8 \%)$, withdrawal of consent $(n=2,8 \%)$, completion of treatment $(n=1,4 \%)$, toxicity (glomerulonephritis, $n=1,4 \%$ ), and death in CRp $(n=1,4 \%)$.

The outcomes in ND patients with 2-year OS of $80 \%$ compare favorably with prior reports of sorafenib, quizartinib, or gilteritinib with LITs which have yielded ORR of $67-92 \%$ and median OS of $8.3-18.6$ month $^{2-4}$. The outcomes in salvage setting with CRc rate of $62 \%$ and median OS of 6.8 months are comparable to nonvenetoclax based doublet regimens of sorafenib, midostaurin, or quizartinib with LITs which have yielded overall response rates (ORR) of $26-83 \%$ and median OS of 5.1-11.3 months ${ }^{1,3}$. In comparison, venetoclax with gilteritinib has shown a CRc of $85 \%$ in $\mathrm{R} / \mathrm{R} F L T 3^{\text {mut }}$ $\mathrm{AML}^{11}$. 


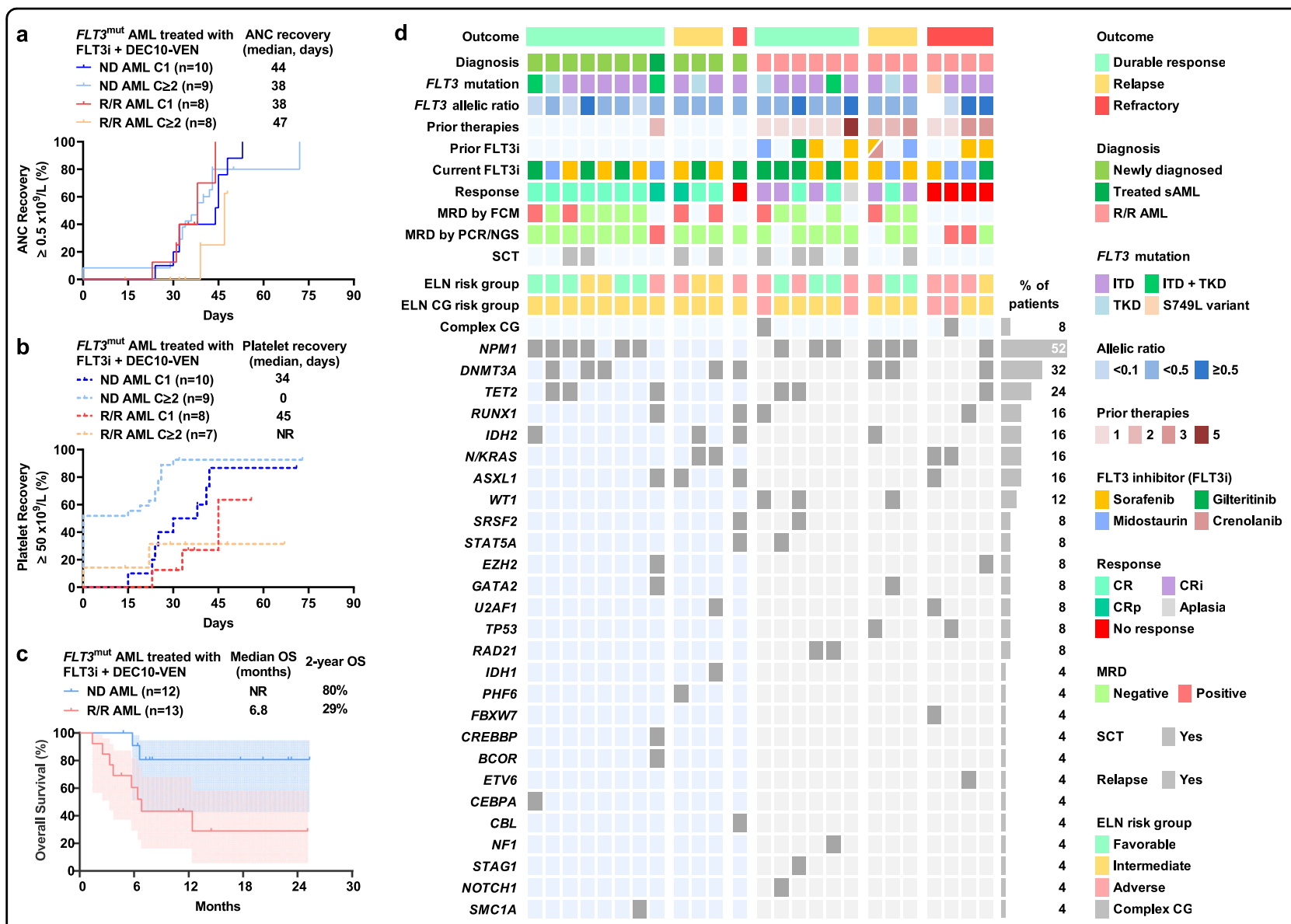

Fig. 1 Outcomes of patients with newly diagnosed (ND) and relapsed/refractory (R/R) acute myeloid leukemia with $F L T 3^{\mathrm{mut}}$ treated with FLT3 inhibitor (FLT3i), venetoclax, and 10-day decitabine (DEC10-VEN). a Absolute neutrophil count (ANC) recovery to $\geq 0.5 \times 10^{9} / \mathrm{L}, \mathbf{b}$ platelet count recovery to $\geq 50 \times 10^{9} / \mathrm{L}$, and $\mathbf{c}$ overall survival (OS), and $\mathbf{d}$ mutational landscape of all patients. NR not reached, MRD measurable residual disease, FCM flow cytometry, multiplex PCR polymerase chain reaction, PCR-based NGS next-generation sequencing.

The rationale for selecting the 10-day regimen over 5days of decitabine as the backbone include previous pharmacodynamic data suggesting better efficacy of the 10 -day regimen and high response rates of $40-64 \%$ in AML with unfavorable risk cytogenetics ${ }^{12,15}$. Although there were delays in blood count recovery, the rates of neutropenic fever in $40 \%$ patients and infections with grade $3 / 4$ neutropenia in $36 \%$ patients were comparable to the $30 \%$ rate of neutropenic fever and $64 \%$ rate of grade $3 /$ 4 infections with azacitidine and venetoclax ${ }^{5}$. Deaths due to infectious etiology occurred in one ND patient (8\%) which was comparable to $7 \%$ infection-related deaths noted with frontline HMA and venetoclax ${ }^{16}$. Hence, without prospective studies, it is difficult to speculate about the risk-benefit ratio of using a 5-day decitabine or 7-day azacitidine regimen as the backbone for such triplet combinations. Future trials need to establish the optimal schedule of venetoclax and FLT3i doublets and triplets to minimize toxicity and maximize efficacy.
For $F L T 3^{\text {mut }}$ patients who are candidate for LITs, we are currently using second-generation FLT3i gilteritinib $80 \mathrm{mg}$ daily, based on similar efficacy to $120 \mathrm{mg}$ dose $^{17}$, and using a cycle 1 day-14 bone marrow to evaluate for response or marrow ablation to determine withholding of venetoclax to promote for earlier ANC recovery in first cycle. This may allow for a longer 'venetoclax holiday' from day-14 onward and potentially improve myelosuppression with such triplet therapy. After achievement of response, we recommend continuous daily dosing of FLT3i and decreasing duration of venetoclax to 14-21 days based on count recovery period in cycle 1 and adding myeloid growth factors as needed to minimize duration of neutropenia. Other trials testing similar triplet combinations of HMA, venetoclax with quizartinib (NCT03661307) and gilteritinib (NCT04140487) in ND and $\mathrm{R} / \mathrm{R}$ AML are currently ongoing and will determine the optimal combinatorial approach for these agents. While the doublet combination of FLT3i with venetoclax 
has shown high CRc rate of $85 \%$ in R/R FLT3 ${ }^{\text {mut }}$ AML, we believe that triplet therapy with the addition of an HMA may offer broader activity and prolong responses and survival by eliminating other subclones and preventing secondary resistance ${ }^{11,18}$. This will need to be balanced against the potential increased myelosuppression with such triplet regimens. Continued accrual and longer follow-up of these trials will hopefully provide more answers and help optimize the selection of the doublets or triplets in specific patient populations.

Some limitations of our study include the use of different FLT3i across different generations which may limit extrapolation of our results. Many patients at our center travel from far locations and choose to receive portion of their care closer to home. Consequently, we could not ascertain causes of deaths in some patients.

In conclusion, triplet therapy with FLT3i, venetoclax, and decitabine is safe and an excellent frontline option for older patients with ND FLT3 ${ }^{\text {mut }}$ AML, and effective for R/ $\mathrm{R}$ AML. Transition to HSCT and post-transplant maintenance with FLT3i may offer further improvement in long-term outcomes.

\section{Acknowledgements}

We would like $t$ thank our patients, their familes and caregivers. A. Maiti. received support through the ASCO Young Investigator Award from the Conquer Cancer Foundation. This study was supported in part by the MD Anderson Cancer Center Support Grant CA016672 from the National Cancer Institute and the Research Project Grant Program (R01CA235622) from the National Institutes of Health.

\section{Author details}

'Department of Leukemia, The University of Texas MD Anderson Cancer Center, Houston, TX, USA. ${ }^{2}$ Division of Pharmacy, The University of Texas MD Anderson Cancer Center, Houston, TX, USA. ${ }^{3}$ Department of Hematopathology, The University of Texas MD Anderson Cancer Center, Houston, TX, USA

\section{Author contributions}

Conception and design: A.M., N.G.D., C.D.D., M.Y.K., and H.M.K. Administrative support: C.D.D., M.Y.K., and H.M.K. Provision of study materials or patients: C.D.D., F.R., N.P., G.B., P.B., G.C.I., T.M.K., N.J.S., M.Y., G.M.B., A.F., E.J.J., N.J., M.O., K.T., P.A.T. K.S.M., S.P., W.G.W., H.M.K., N.G.D., and M.Y.K. Collection and assembly of data: K.S.M., S.P., and A.M. Data analysis and interpretation: A.M., C.D.D., C.R.R., N.G.D., and M.Y.K. Manuscript writing: A.M., C.D.D., N.G.D., and M.Y.K. Critical revision for important intellectual content: All authors. Final approval of manuscript: All authors reviewed and approved the final version of the manuscript.

\section{Competing interests}

A.M. received research funding from Celgene Corporation. C.D.D. reports personal fees from Abbvie, Agios, Novartis, ImmuneOnc, Daiichi-Sankyo, Celgene, Jazz, and Notable Labs, outside the submitted work. N.G.D. reports grants from Abbvie, Genentech, Astellas, Daiichi-Sankyo, Pfizer, BMS, Immunogen, Novimmune, Forty Seven, Inc., personal fees from Abbvie, Genentech, Astellas, Daiichi-Sankyo, Pfizer, BMS, Immunogen, Jazz Pharmaceuticals, Trillium, Forty Seven, Inc., Gilead, Kite, and Novartis. C.R.R.: none. F.R. received personal fees and research grants from Abbvie. N.P. reports personal fees from Pacylex Pharmaceuticals, Incyte, LFB Biotechnologies, Roche Diagnostics, and Blueprint Medicines, grants and other from Affymetrix, grants from SagerStrong Foundation, personal fees and other from Novartis, personal fees, nonfinancial support, and other from Stemline Therapeutics and AbbVie, personal fees and nonfinancial support from Celgene, MustangBio, and DAVA Oncology, and other from Samus Therapeutics, Cellectis, Daiichi-
Sankyo, and Plexxikon, outside the submitted work. G.B.: research fundingAbbVie, Incyte, Janssen, GSK, and Cyclacel; consultancy and research funding -BioLine Rx; consultancy—NKarta and PTC Therapeutics; research fundingOncoceutics, Inc. M.O. and G.M.B: none. N.J.S. reports grants from Takeda Oncology and Astellas; personal fees from Takeda Oncology, AstraZeneca, and Amgen. Y.A. and T.M.K.: none. K.T. has received personal fees for service on the advisory boards of Symbio Pharmaceuticals, GSK, and Celgene. M.Y.: none. N.J.: consultancy, honoraria, membership on an entity's Board of Directors or advisory committees, and research funding-AbbVie, Pharmacyclics, an AbbVie company, Janssen Pharmaceuticals, Inc., Genentech, Pfizer, ADC Therapeutics, AstraZeneca, Servier, Verastem, Precision Biosciences, and Adaptive Biotechnologies; research funding - BMS, Incyte, and Cellectis. P.B. reports research grant and personal fees from Incyte, Celgene, CTI Biopharma, Kartos Therapeutics, and BluePrint Medicines, and research grants from Constellation Pharmaceuticals, NS Pharma, Promedior, Pfizer, and Astellas. A.F.: none. G.C.I. received research funding from Celgene and served on an advisory board for Novartis. E.J.J.: consultancy research funding from Takeda, BMS, Adaptive, Amgen, AbbVie, Pfizer, and Cyclacel LTD; research grants with Amgen, Abbvie, Spectrum, BMS, Takeda, Pfizer, and Adaptive. P.A.T. reports research funding to the institution and consultancy/honorarium paid to the institution from Pharmacyclics, Abbvie, and Amgen; consultancy/honorarium paid to the institution from Genentech, Gilead Scienes, and Janssen-Cilag; research funding to the institution from Pfizer. K.S.M., S.A.P., and W.G.W.: none. H.M.K.: grants and other from AbbVie, Agios, Amgen, Immunogen, and Pfizer; grants from Ariad, Astex, BMS, Cyclacel, Daiichi-Sankyo, Jazz Pharma, Novartis, Actinium, and Takeda, outside the submitted work. M.Y.K. has received grants from NIH, NCl, Abbvie, Genentech, Stemline Therapeutics, Forty Seven, Inc., Eli Lilly, Cellectis, Calithera, Ablynx, and AstraZeneca; consulting/honorarium from AbbVie, Genentech, F. Hoffman La-Roche, Stemline Therapeutics, Amgen, Forty Seven, Inc., and Kisoji; clinical trial support from Ascentage; stocks/royalties in Reata Pharmaceutical.

\section{Publisher's note}

Springer Nature remains neutral with regard to jurisdictional claims in published maps and institutional affiliations.

Supplementary information The online version contains supplementary material available at https://doi.org/10.1038/s41408-021-00410-w.

Received: 5 September 2020 Revised: 27 October 2020 Accepted: 5 November 2020

Published online: 01 February 2021

\section{References}

1. Wang, E. S. Incorporating FLT3 inhibitors in the frontline treatment of FLT3 mutant acute myeloid leukemia. Best Pract. Res. Clin. Haematol. 32, 154-162 (2019).

2. Ohanian, M. et al. Sorafenib combined with 5-azacytidine in older patients with untreated FLT3-ITD mutated acute myeloid leukemia. Am. J. Hematol. 93, 1136-1141 (2018).

3. Swaminathan, $\mathrm{M}$. et al. The combination of quizartinib with azacitidine or low dose cytarabine is highly active in patients (Pts) with FLT3-ITD mutated myeloid leukemias: interim report of a phase I/II trial. Blood 130, 723-723 (2017).

4. Esteve, J., Schots, R. \& Castillo, T. Gilteritinib plus azacitidine in newly diagnosed FLT3 mutated (FLT3mut.) acute myeloid leukaemia patients ineligible for intensive induction chemotherapy: preliminary findings from the safety cohort. EMJ Hematol 7, 50-52 (2019).

5. DiNardo, C. D. et al. Azacitidine and venetoclax in previously untreated acute myeloid leukemia. N. Engl. J. Med. 383, 617-629 (2020).

6. $\mathrm{Ma}$, J. et al. Inhibition of $\mathrm{BCl}-2$ synergistically enhances the antileukemic activity of midostaurin and gilteritinib in preclinical models of FLT3-mutated acute myeloid leukemia. Clin. Cancer Res. 25, 6815-6826 (2019).

7. Mali, R. S. et al. Venetoclax combines synergistically with FLT3 inhibition to effectively target leukemic cells in FLT3-ITD+ acute myeloid leukemia models. Haematologica https://doi.org/10.3324/haematol.2019.244020 (2020).

8. Chyla, B. et al. Correlative biomarkers of response to venetoclax in combination with chemotherapy or hypomethylating agents in elderly untreated patients with acute myeloid leukemia. Blood 128, 1709-1709 (2016). 
9. Rahmani, M. et al. Inhibition of Bcl-2 antiapoptotic members by obatoclax potently enhances sorafenib-induced apoptosis in human myeloid leukemia cells through a Bim-dependent process. Blood 119, 6089-6098 (2012).

10. Ravandi, F. et al. Phase 2 study of azacytidine plus sorafenib in patients with acute myeloid leukemia and FLT-3 internal tandem duplication mutation. Blood 121, 4655-4662 (2013).

11. Perl, A. E. et al. Venetoclax in combination with gilteritinib in patients with relapsed/refractory acute myeloid leukemia: a phase $1 \mathrm{~b}$ study. Blood 134, 3910-3910 (2019).

12. DiNardo, C. D. et al. 10-day decitabine with venetoclax for newly diagnosed intensive chemotherapy ineligible, and relapsed or refractory acute myeloid leukaemia: a single-centre, phase 2 trial. Lancet Haematol $\mathbf{1 3}$ e724-e736 (2020).

13. Perl, A. E. et al. Gilteritinib or chemotherapy for relapsed or refractory FLT3mutated AML. N. Engl. J. Med. 381, 1728-1740 (2019).
14. Cortes, J. E. et al. Quizartinib versus salvage chemotherapy in relapsed or refractory FLT3-ITD acute myeloid leukaemia (QUANTUM-R): a multicentre, randomised, controlled, open-label, phase 3 trial. Lancet Oncol. 20, 984-997 (2019).

15. Issa, J.-P. J. et al. Phase 1 study of low-dose prolonged exposure schedules of the hypomethylating agent 5-aza-2'-deoxycytidine (decitabine) in hematopoietic malignancies. Blood 103, 1635-1640 (2004).

16. DiNardo, C. D. et al. Updated safety and efficacy of venetoclax with decitabine or azacitidine in treatment-naive, elderly patients with acute myeloid leukemia. Blood 130, 2628-2628 (2017).

17. Perl, A. E. et al. Final results of the chrysalis trial: a first-in-human phase $1 / 2$ dose-escalation, dose-expansion study of gilteritinib (ASP2215) in patients with relapsed/refractory acute myeloid leukemia (R/R AML). Blood 128, 1069-1069 (2016).

18. Short, N. J. et al. Advances in the treatment of acute myeloid leukemia: new drugs and new challenges. Cancer Discov. 10, 506-525 (2020). 